NANO: Brief Reports and Reviews

Vol. 13, No. 10 (2018) 1850120 (9 pages)

(C) World Scientific Publishing Company

DOI: $10.1142 /$ S1793292018501205

\title{
Control of Wettability Using Regularly Ordered Two-Dimensional Polymeric Wavy Substrates
}

\author{
Dong Kee Yi \\ Department of Chemistry, Myongji University \\ Myonji-ro 116, Yongin, Gyeonggi-do, Republic of Korea \\ vitalis@mju.ac.kr
}

Received 30 July 2018

Accepted 10 September 2018

Published 12 October 2018

\begin{abstract}
Two-dimensional poly(dimethylsiloxane) (PDMS) films with wavy patterns were studied in order to investigate reversible and irreversible wetting effects. Pre-strained, surface oxidized layers of PDMS were used to form relieved wavy geometries, on which hydrophobic functionalization was carried out in order to produce irreversible wetting effects. Wavy-patterned PDMS films showed time-dependent reversible wetting effects. The degree of surface wettability could be tuned by the choice of wavy groove geometries. And the groove geometries were controlled via $\mathrm{O}_{2}$ plasma treatment and mechanical pre-straining. The pre-strained, buckled PDMS films were applied to the fabrication of hydrophobic polystyrene nano-patterns using colloidal self-assembly, where the colloids were arrayed in two-dimensional way. The wavy polystyrene films were found to be more hydrophobic relative to flat polystyrene films. The grooving methodology used in this study could be applied to enhancing the hydrophobicity of other types of polymeric thin films, eliminating the need for chemical treatment.
\end{abstract}

Keywords: Wavy polymer film; wettability control; colloidal self assembly; PDMS thin film; reversible wetting; irreversible wetting; delaminated film.

\section{Introduction}

Controlling the wettability of polymeric substrates is of great importance in the development of flexible, highly functional materials with applications in the arraying and ordering of biological cells, ${ }^{1}$ the formation of hydrophobic nanofilms for wearable devices, ${ }^{2-4}$ the preparation of regenerative cell scaffolds, ${ }^{5}$ water repellent fabrics ${ }^{6}$ and the creation of artificial gecko's feet for self-cleaning adhesives. ${ }^{7}$ The hydrophilic/hydrophobic property of a surface is quantified by the contact angle (CA) formed by a water droplet cast on the surface. ${ }^{8}$ Therefore, controlling of the wetting property is monitored by measuring the CA. ${ }^{9,10}$ Formation of hydrophobic surfaces has been pursued via (1) treatment with low-surface energy chemicals such as fluorinated or silicon-containing compounds, and (2) creation of nano/micro structures on the surface. Specifically, linear nanowire arraying ${ }^{7,11}$ and particulate nanomaterial coating can form super-hydrophobic surfaces. ${ }^{12,13}$ In contrast, an assembly of thermo- and photo-responsive molecules on the surface can be used as an outer stimulus to induce tunable hydrophilic/hydrophobic surface properties. ${ }^{14-16}$ Even though, no perfect consensus exists within the surface science community, ${ }^{17}$ we assume herewith that the surface hydrophobicity for a nanostructured 
substrate can be explained by Wenzel's model, where the droplet completely fills the depressions in the nanopatterned surface over the projected area of surface-water contact, ${ }^{18}$ and by Cassie's model, where the water does not fill the concave areas of the nanopatterned surface but rests on a convective area composed of the solid material and air. ${ }^{19}$

Flexible substrates with poly(dimethylsiloxane) (PDMS) are gaining increasing importance in various engineering applications such as flexible antireflective nanostructures,${ }^{20}$ patterned cell arrays, ${ }^{1}$ bioelectromechanical systems, ${ }^{21}$ and high-performance electronics. ${ }^{22,23}$ PDMS has advantages in structural fabrication over silicon because of low cost for prototyping, fast processing, and simplified preparation steps. PDMS nanoarchitectures have been studied using soft lithographic methods. ${ }^{24}$ Early-on, Whitesides et $a l .{ }^{25,26}$ pursued studies on thin metal films grown onto a PDMS substrate, which showed that sub 100-nm thin metallic or oxide films can be distorted to form a wavy pattern onto a flexible polymer substrate at room temperature.

To our knowledge, two-dimensionally ordered polymeric wavy patterns onto a PDMS substrate has not yet been applied to induce controlled wettability. Since wavy patterning onto a PDMS is (1) a simple step process, (2) applicable to wide-area fabrication (wafer scale), (3) of low cost, (4) ecofriendly, not requiring hydrophobic, toxic chemicals or fluorinated compounds, and (5) amenable to versatile use, we decided to study how surface wettability could be controlled by surface topography variation. Herein, we report on the wettability control of two wavy polymeric substrates: (1) PDMS and (2) polystyrene.

\section{Experimental Details}

\subsection{PDMS thin film preparation and characterization}

PDMS (0.3 mm thickness, Sylgard 184, Dow Corning, base to cross-linking agent mass ratio $10: 1$, curing at $80^{\circ} \mathrm{C}$ for $20 \mathrm{~h}$ ) film was mechanically prestrained using a homemade equipment from $1 \%$ to $30 \%$, and the strain direction was uniaxial. Prestrained PDMS was treated with oxygen plasma $(150 \mathrm{~W})$ for 1 to $5 \mathrm{~min}$ and then released to form two-dimensional wavy patterns [see Fig. 1(a)]. The resulting dimensions of wavy nanopatterns were analyzed using atomic force microscopy (AFM, DI) in the noncontact mode. For comparison, nostrained PDMS was also treated with oxygen plasma $(150 \mathrm{~W})$.

For imparting hydrophobic properties by chemical means, $100 \mu \mathrm{L}$ of octadecyl-tri-methoxysilane ethanol solution was vacuum-evaporated for two hours at room temperature and deposited onto the pre-strained PDMS substrate (1-30\%, and 5 min $\mathrm{O}_{2}$ plasma treated) in a desiccator. Subsequently, the pre-strained PDMS samples were released to form wavy patterns.

\subsection{PS thin film preparation onto the PDMS via a convective arraying method}

Mono-layered PS colloid crystals were fabricated by the method of confined convective assembly, previously reported by us in detail. ${ }^{27-30}$ Briefly, we used an aqueous suspension (about $50 \mu \mathrm{L}$ ) of $495 \mathrm{~nm}$ diameter PS colloidal particles (Thermofisher) of

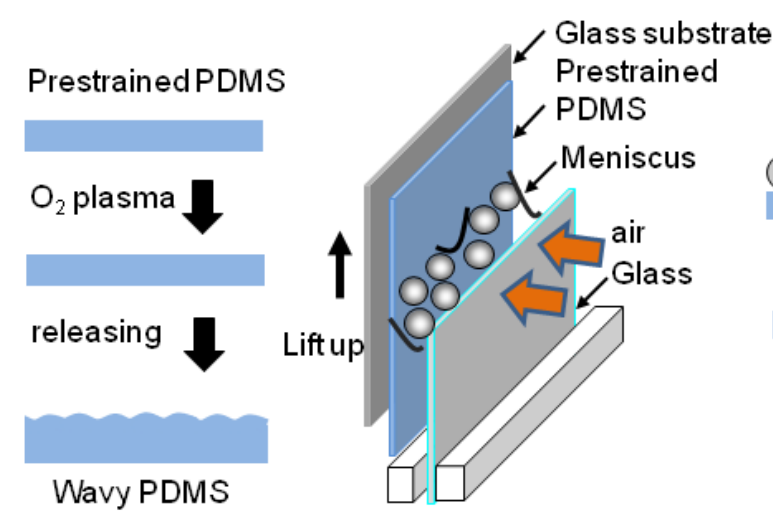

(a)

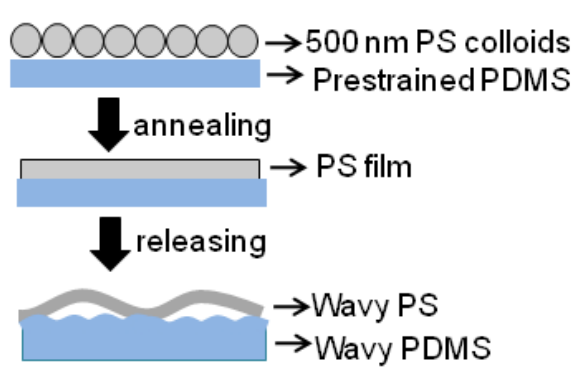

(c)

Fig. 1. Schematic diagrams of the procedure followed for the fabrication of wavy PDMS films (a) and wavy polystyrene films (b,c). 
1.0 wt.\% concentration. The colloidal suspension was inserted into the gap of the front pre-strained PDMS substrate (fixed substrate) and the back glass substrate (moving substrate) in the confined convective assembly setup [see Fig. 1(b)]. The glass and pre-strained PDMS substrates had been treated with oxygen plasma for $1 \mathrm{~min}$ just prior to this procedure in order to increase the wettability of the film to aqueous colloidal dispersions. Subsequently, the back substrate was raised at a rate of $30 \mu \mathrm{m} / \mathrm{s}$ as air-flux was added towards the meniscus formed at the interface of the substrates and the colloidal suspension. The colloidal PS particles then assembled into a monolayer on the pre-strained PDMS substrate.

The colloidal polystyrene film covered about $40 \%$ of the PDMS surface area because the margins of the PDMS film were covered by a spacer that created a gap between the PDMS and the glass substrate. The resulting monolayer polystyrene colloid placed on the pre-strained PDMS was heated at $160^{\circ} \mathrm{C}$ for $12 \mathrm{~min}$ in a convection oven. This thermal aging induced a flat polystyrene thin film. Meanwhile, the strained PDMS substrate was released to induce a wavy polystyrene pattern mounted on the PDMS at room temperature [see Fig. 1(c)].

\subsection{Contact angle measurement and surface topography analysis}

CAs were measured using a CA analyzer (Phoenix 300, SEO, Korea). A sessile pure distilled water drop, $10 \mu \mathrm{L}$, was cast onto a nanopatterned wavy substrate in air and the drop image was captured by a CCD camera interfaced with the CA analyzer and recorded. The CA of 10 drops were measured through the recorded images and averaged. Noncontact mode probes were used for AFM analysis.

\section{Results and Discussion}

For CAs less than $10^{\circ}$, the surface is called superhydrophilic; less than $90^{\circ}$ hydrophilic; larger than $90^{\circ}$ but less than $150^{\circ}$ hydrophobic; and for CAs larger than $150^{\circ}$ super-hydrophobic. The averaged water drop CA values and corresponding droplet images on the PDMS films, $\mathrm{O}_{2}$ plasma treated for 5 min, can be found in Fig. 2.

When the CA was measured immediately after releasing the pre-strained film, 1-30\% PDMS with hydrophilic nature was observed $\left(\mathrm{CA}<90^{\circ}\right)$, while

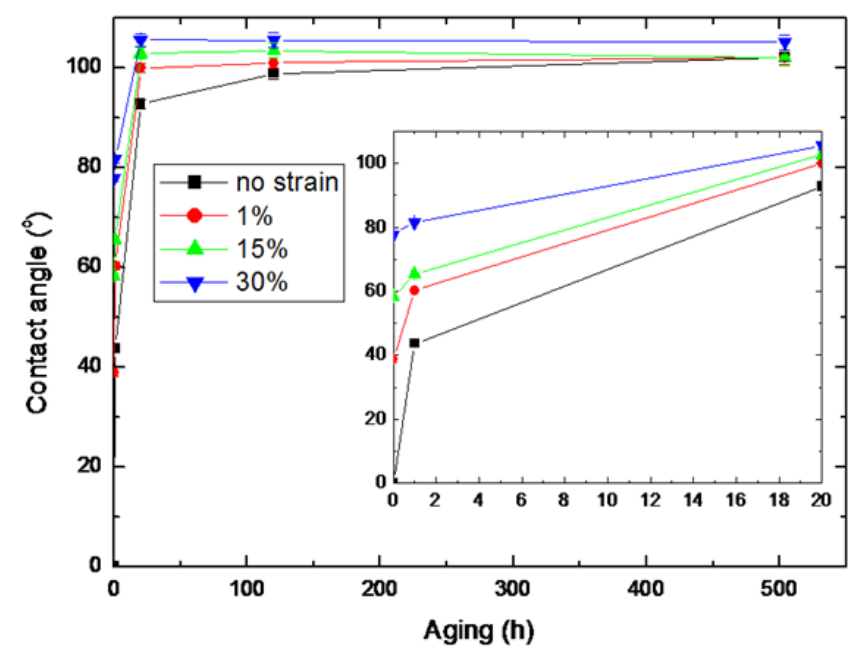

(a)

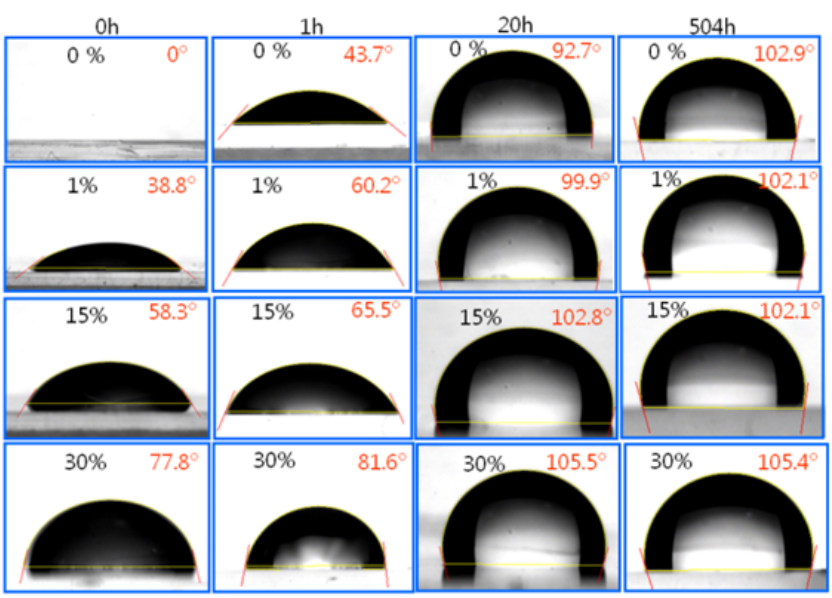

(b)

Fig. 2. (a) Water droplet contact angle values on the wavy PDMS films, with different pre-strain values as indicated, as a function of aging (inset for enlarged view). (b) Corresponding droplet images are shown at different aging times.

Note: Pre-strained PDMS was treated with $\mathrm{O}_{2}$ plasma for $5 \mathrm{~min}$.

in the case of nonstrained PDMS, super-hydrophilic nature was observed $\left(\mathrm{CA}<10^{\circ}\right)$ due to surface oxidation by $\mathrm{O}_{2}$ plasma treatment. All PDMS samples were kept within a clean room, ISO 5 class; they were aged to a maximum of 504 hs for timedependent CA measurement. By $120 \mathrm{~h}$ aging, the $\mathrm{CA}$ value had reached a saturated value for most strained PDMS samples. At the full aging stage of $504 \mathrm{hs}$ nonstrained and strained PDMS showed no significant differences.

The data indicated that the CA increases linearly with aging time up to a saturation level, as shown in Fig. 2(a), and enlarged view in the inset. Dependency 
of $\mathrm{CA}$ values on strain was prominent at the initial stage but faded away with aging. It was, therefore, concluded that PDMS substrate hydrophilicity can be controlled at the early stage, prior to saturation, by varying the mechanical strain. Conventionally, control of surface wettability has been achieved by one of two methods: the creation of a geometrical architecture on the surface or the chemical modification of the surface. In our study, PDMS films were exposed to $\mathrm{O}_{2}$ plasma environment, were mechanically stressed uniaxially, and then released. Literature reveals that $\mathrm{O}_{2}$ plasma can induce surface oxidation of $\mathrm{PDMS},{ }^{31}$ and that a regular order of buckling can be fabricated to relieve compressive energy in the upper most top layer of PDMS via thermally induced mechanical strains. ${ }^{31}$ Figure 3 shows the evidence of wavy patterns on the pre-strained PDMS substrates. In our experimental conditions, no trace of wavy geometry was observed from nonstrained PDMS films through the AFM topography analysis. Pre-strain conditions determine the buckling periodicity, wavelength, buckling depth and amplitude. The observed wavelength was disproportional to the applied strain but the amplitude was proportional to the strain [see Fig. 3(a)].

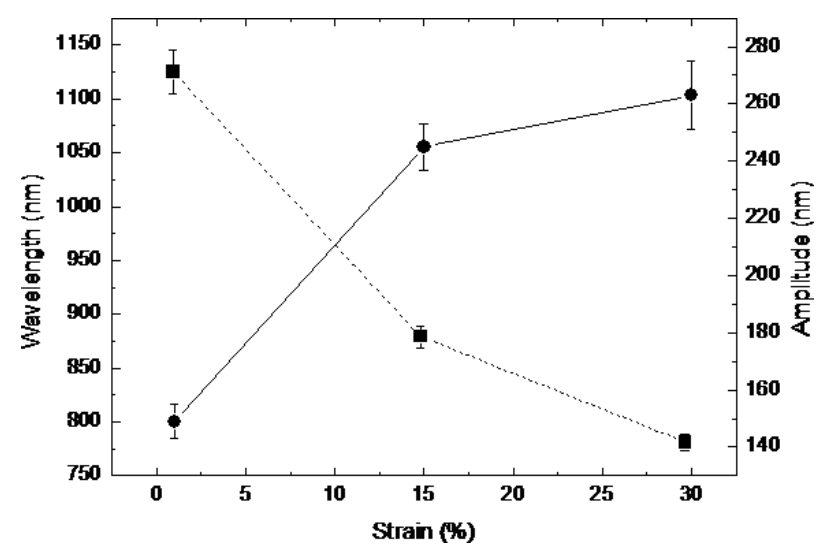

(a)

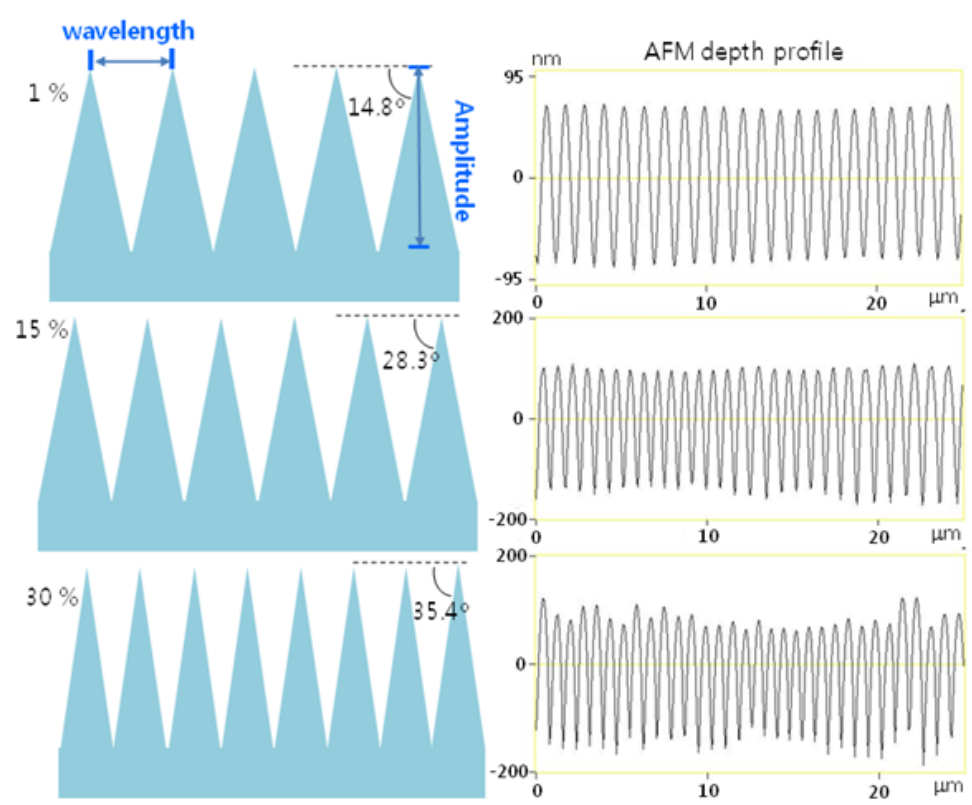

(b)

Fig. 3. (a) Dependence of amplitude (solid line) and wavelength (dotted line) values on strain for the wavy PDMS films. (b) Schematic models of wavy geometries (left column), and the corresponding AFM depth profile data (right column) of the resulting wavy PDMS films. 
As noted earlier, the wettability of a solid surface can be controlled by the introduction of geometrical patterns or nanoarchitecture on the surface, while hydrophobicity can be enhanced by creating a local geometry with a large relative to the projected geometrical area such as pillar arrays. ${ }^{7}$ This topography increases hydrophobicity in accord with Wenzel's and Cassie-Baxter's models. ${ }^{18,19}$ According to the AFM data, we can propose schematic models for the pre-strain dependent wavy patterns on the PDMS films [see Fig. 3(b)]. The drawings, on the left column of Fig. 3(b), show how the convective grooves protrude, and how the degree of slope of the grooves depends on different pre-strains. The right column of Fig. 3(b) shows the corresponding AFM depth profiles in the regions where regularly ordered grooves were observed.

In accord with the Wenzel model, relatively lower height and slope groove geometry, caused by low pre-strain, led to more hydrophilic behavior due to the ease of water spreading in the concave groove areas; however, higher pre-strain-induced higher protrusion and slope of wavy geometries and resulted in difficulty of water spreading on the concave groove areas (Cassie-Baxter conditions). Consequently, more hydrophobic surface property was realized; higher water droplet $\mathrm{CA}$. Although some criticism has been raised against Wenzel's and Cassie-Baxter's model,${ }^{17}$ where contact area plays

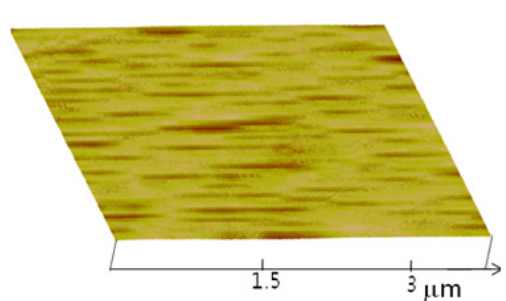

(a)

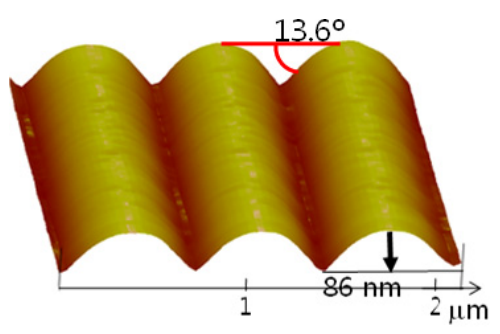

(c) an important role in wettability, our results can be rationalized on the basis of Wenzel's and CassieBaxter's model.

In addition to surface topography, surface chemistry also affects the wettability of a solid surface. The oxidized nature of the uppermost thin layer of the PDMS film imparts high hydrophilic property to the PDMS surface and resulted in broad spread of a water droplet at an early stage [see Fig. 2(b)]. But the oxidation process does not seem to be irreversible because the PDMS hydrophobicity increases with time. When the PDMS surface was measured after full aging, $504 \mathrm{hs,} \mathrm{no} \mathrm{dimensional}$ variation was observed in the AFM topography data. This means that the observed increment in hydrophobicity with time was caused by the reversible PDMS surface chemistry. In-depth study of irreversible chemical treatment of PDMS will be discussed later.

It was also found that $\mathrm{O}_{2}$ plasma treatment for various time spans, $1-5 \mathrm{~min}$, can induce the formation of different wavy geometries when $1 \%$ prestrain was applied to all samples. It was observed that less than 2-min exposure to $\mathrm{O}_{2}$ plasma did not induce prominent wavy patterns on the PDMS; however, $3 \mathrm{~min}, 4 \mathrm{~min}$, and $5 \mathrm{~min}$ treatment resulted in a proportional increase of both the wavelength and the amplitude of the wavy structures (see Fig. 4). Water droplet CA data, collected

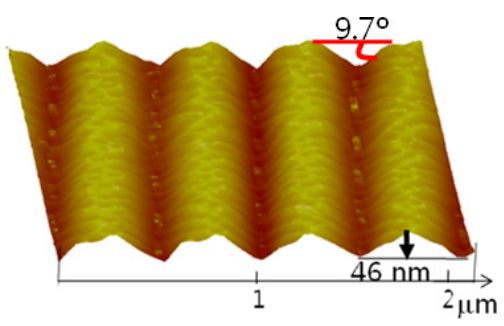

(b)

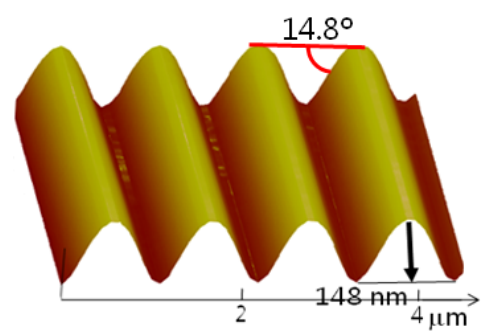

(d)

Fig. 4. Dependence of PDMS-film wavy geometries on $\mathrm{O}_{2}$ plasma treatment time for specimen prepared with $1 \%$ pre-strain. AFM depth profile data, (a) treatment time less than $2 \mathrm{~min}$, (b) $3 \mathrm{~min}$, (c) $4 \mathrm{~min}$ and (d) $5 \mathrm{~min}$. 


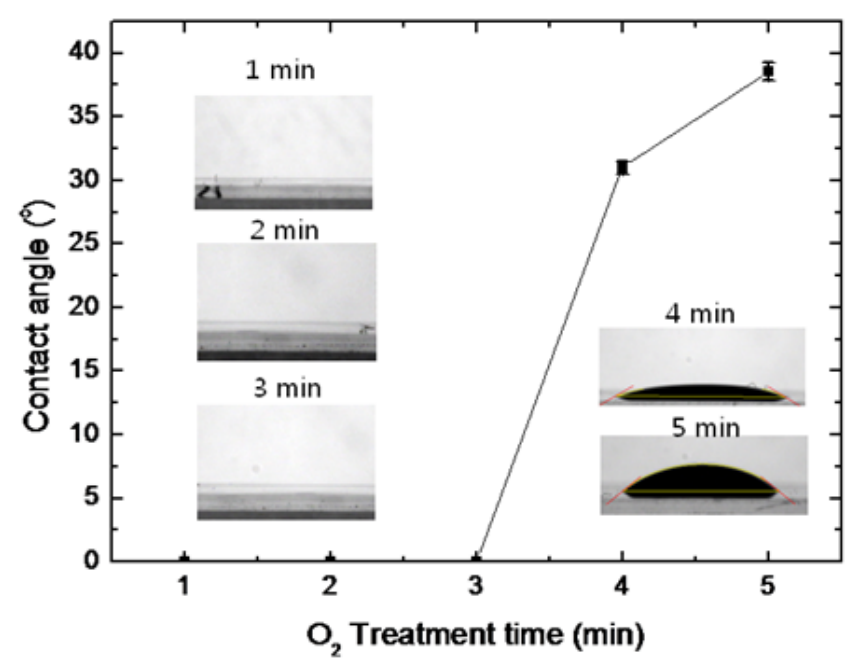

Fig. 5. Dependence of water droplet contact angle values on the wavy PDMS film on $\mathrm{O}_{2}$ plasma treatment time, all specimen prepared with $1 \%$ pre-strain (inset: droplet images at $1-5 \mathrm{~min})$.

immediately after the fabrication of the PDMS patterns, indicated that weak surface protrusion, caused by $1 \mathrm{~min}, 2 \mathrm{~min}$, and 3 -min treatment, exhibited super-hydrophilic wettability (Fig. 5). It must be noted that although a 3-min treatment induced measurable protrusion on the PDMS surface [Fig. 4(b)], water droplets were well spread. In the case of 5-min treatment, the resulting wavy PDMS geometries and CA value, $c a .38^{\circ}$, were quite comparable to those of a previous $1 \%$ straindependent study [see Fig. 2(b)], indicating consistency in the performance of the studies.

The irreversible surface wettability via chemical treatment was further studied by using an active octadecyl-tri-methoxysilane possessing a long hydrophobic chain conjugated with silanol, Si-OH, groups. Basic ethanol solution, $\mathrm{pH} \sim 10$, catalyzed methoxy functions into the silanol groups, while the hydroxyl groups reacted with the oxidized silane on the pre-strained PDMS surface via covalent bond formation. Water droplet CA measurement was carried onto the octadecyl-tri-methoxysilane-treated PDMS surfaces. Interestingly, CA values were stabilized with aging time and showed strain dependency [Fig. 6(a)]. The observed wavy pattern [Fig. 6(b)] showed similar wavelength and amplitude as compared to the nonchemically treated case [Fig. 3(a)], i.e., only geometrical contribution. In comparison with the nonchemically treated cases (see Fig. 2), strain effects on the resulting CA were clear and dependence on aging time were clear for

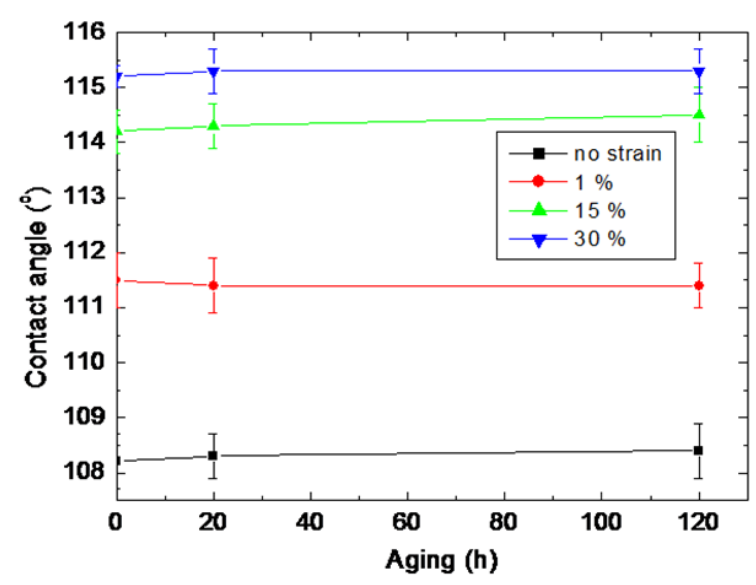

(a)

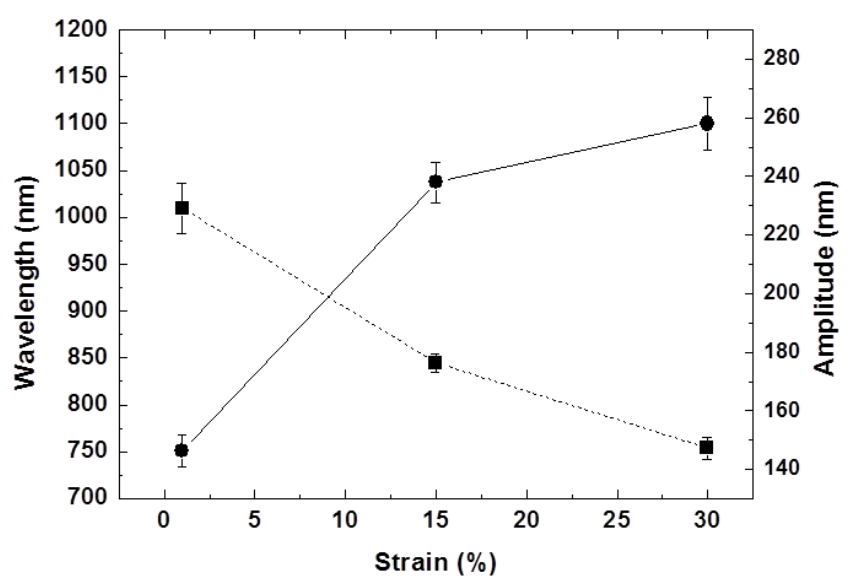

(b)

Fig. 6. (a) Water droplet contact angle values at different aging times and (b) amplitude (solid line) and wavelength (dotted line) values of octadecyl-tri-methoxysilane-treated wavy PDMS at different applied strains (1-30\% pre-strained).

high strain cases, $15 \%$ and $30 \%$, with $\mathrm{CA}$ values increasing with aging.

We speculate that covalent bonding between the PDMS and octadecyl-tri-methoxysilane caused irreversible hydrophobicity. Furthermore, this early formation of strong covalent bonds also affects the dependence of the CA on strain even at the fullyaged stage.

In our study, we raised the question whether it would be feasible to apply these wavy PDMS films as templates for fabricating other types of hydrophobic polymer films. To address this question, we followed our previously developed convective colloidal array approaches, where a monolayer of colloidal polystyrene was arrayed onto a substrate. ${ }^{27-29}$ Figures 1(b)-1(c) shows the schematic flow of the 

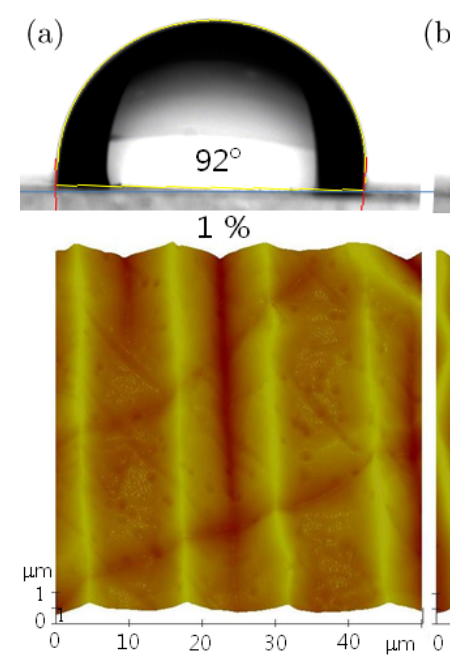

(c)

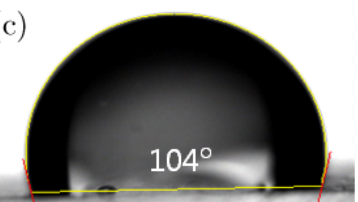

$30 \%$

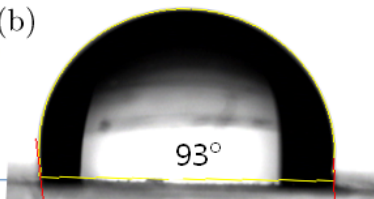

$15 \%$
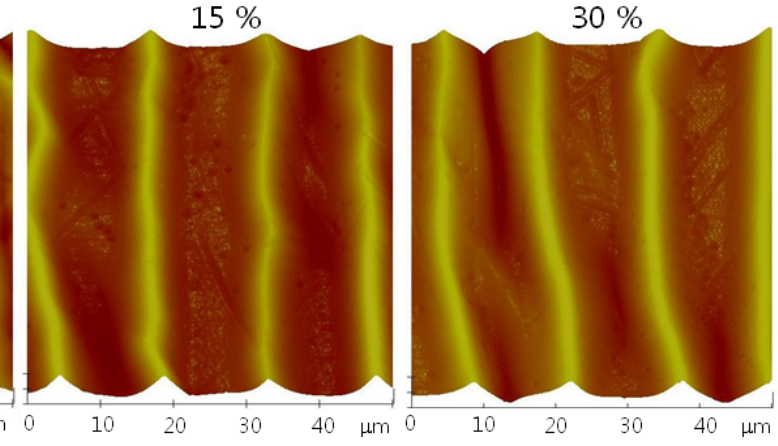

Fig. 7. Water droplet image and contact angle value (upper part) and the corresponding wavy PS film geometries by AFM measurement (lower part), using (a) 1\%, (b) 15\%, and (c) 30\% pre-strained PDMS substrate.

experimental procedure in brief. During the arraying process, the colloid-colloid densification proceeded via lateral capillary forces leading to the formation of a compact array. Subsequent thermal annealing, at $160^{\circ} \mathrm{C}$ induced flat, even polystyrene thin films, $\sim 170 \mathrm{~nm}$ thick, onto both nonstrained and pre-strained (1-30\%) PDMS substrates. Releasing the pre-strained PDMS films induced the formation of buckled polystyrene wavy patterns. The buckled mode did not wrinkle, but delaminated. AFM topographic images, shown in the lower part of Fig. 7, indicate that the polystyrene wavy pattern dimensions are quite different from those of the PDMS wavy pattern, as seen in Figs. 3 and 4. In case of polystyrene the wavy groove depth, $\sim 740 \mathrm{~nm}$ for $1 \%, 1400 \mathrm{~nm}$ for $15 \%, 1700 \mathrm{~nm}$ for $30 \%$, and periodicity, $\sim 13.5 \mu \mathrm{m}$ for all strain conditions, showed highly increased values compared to those of PDMS. This means that when the pre-strained PDMS substrate is released, the top-polystyrene film detaches from the bottom PDMS substrate and deforms via a buckle delamination mode to form wavy patterns (see Fig. S1); one expects Poisson effects to lead to compressive strains in the orthogonal direction with a magnitude of about half of that in the direction of applied strain. The resulting polystyrene wavy patterns exhibited increased hydrophobicity, larger water CA $\sim 92-104^{\circ}$ (upper part of Fig. 7), compared to the case of nonstrained flat polystyrene $\sim 83^{\circ}$ (see Fig. S2). The observed water $\mathrm{CA}$ of $\sim 83^{\circ}$ on a flat polystyrene substrate is in good agreement with literature values of 80-86 ${ }^{\circ} .32$ Furthermore, this CA value was not reversible with time up to $504 \mathrm{hs}$. We speculate that the observed increase in hydrophobicity is attributed to the wavy morphology of the polystyrene film; such speculation is fully supported and understood by both Wenzel's and Cassie-Baxter's models. We believe that the polymeric wavy thinfilm fabrication method described herein could be applied broadly to the enhancement of hydrophobicity of other types of polymeric thin films, thus, eliminating the need for chemical treatment.

\section{Conclusions}

The surface wettability of wavy-patterned PDMS thin films was tuned by the details of the grooved geometries on the surface of the films. The grooved geometrical nanoarchitectures were controlled via the application of various pre-strain conditions and $\mathrm{O}_{2}$ plasma treatment time duration. The wavy PDMS films exhibited time-dependent reversible wettability, which was understood to be the result of the reversible surface chemistry of PDMS. In the case of the chemically treated wavy surface of PDMS, saturated CA values were observed for the entire time of the experimental. The buckling nature of pre-strained PDMS was further applied to the investigation of the dependency of surface hydrophobicity on different geometries of wavy polystyrene thin films, which were fabricated using colloidal self-assembly. The polystyrene nanothinfilm did not wrinkle, but delaminated to form wavy patterns exhibiting increased hydrophobicity compared to the nonstrained flat polystyrene. 


\section{Acknowledgments}

This research was supported by Korean NRF, 2015R1D1A1A01058263 and the Korea Institute of Energy Technology Evaluation and Planning (KETEP), and the Ministry of Trade, Industry \& Energy (MOTIE) of the Republic of Korea (No. 20174010201160). DKY thanks Prof. G. C. Papaefthymiou for valuable discussion.

\section{References}

1. D. K. Yi, M. J. Kim, L. Turner, K. S. Breuer and D.-Y. Kim, Biotech Letters 28, 169 (2006).

2. L. Feng, S. Li, H. Li, J. Zhai, Y. Song, L. Jiang and D. Zhu, Angew. Chem., Int. Ed. 41, 1221 (2002).

3. L. Li, Y. Bai, L. Li, S. Wang and T. A. Zhang, Adv. Mater. 29, 1702517 (2017).

4. V. Pandiyarasan, S. Suhasini, J. Archana, M. Navaneethan, A. Majumdar, Y. Hayakawa and H. Ikeda, Appl. Surf. Sci. 418, 352 (2017).

5. Y. Wang, E. Bella, C. S. D. Lee, C. Migliaresi, L. Pelcastre, Z. Schwartz, B. D. Boyan and A. Motta, Biomaterials 31, 4672 (2010).

6. T. Zhu, C. Cai, J. Guo, R. Wang, N. Zhao and J. Xu, ACS Appl. Mater. Interfaces 9, 10224 (2017).

7. T. S. Kustandi, V. D. Samper, D. K. Yi, W. S. Ng, P. Neuzil and W. Sun, Adv. Funct. Mater. 17, 2211 (2007).

8. X.-M. Li, D. Reinhoudt and M. Crego-Calama, Chem. Soc. Rev. 36, 1350 (2007).

9. J. P. Youngblood and T. J. McCarthy, Macromolecules 32, 6800 (1999).

10. W. Chen, A. Y. Fadeev, M. C. Hsieh, D. O. Ner, O. Ner, J. O. Ner and T. J. McCarthy, Langmuir 15, 3395 (1999).

11. K. K. S. Lau, J. Bico, K. B. K. Teo, M. Chhowalla, G. A. J. Amaratunga, W. I. Milne, G. H. McKinley and K. K. Gleason, Nano Lett. 3, 1701 (2003).

12. J. Hong, W. K. Bae, H. Lee, S. Oh, K. Char, F. Caruso and J. Cho, Adv. Mater. 19, 4364 (2007).

13. G. Zhang, D. Y. Wang, Z. Z. Gu and H. Mohwald, Langmuir 21, 9143 (2005).
14. T. Sun, G. Wang, L. Feng, B. Liu, Y. Ma, L. Jiang and D. Zhu, Angew. Chem. Int. Ed. 43, 357 (2004) 357.

15. S. Wang, H. Liu, D. Liu, X. Ma, X. Fang and L. Jiang, Angew. Chem. Int. Ed. 46, 3915 (2007).

16. J. T. Han, D. H. Lee, C. Y. Ryu and K. Cho, J. Am. Chem. Soc. 126, 4796 (2004).

17. L. Gao and T. J. McCarthy, Langmuir 23, 3762 (2007).

18. R. N. Wenzel, Ind. Eng. Chem. 28, 988 (1936).

19. A. B. D. Cassie and S. Baxter, Trans. Faraday. Soc. 40, 546 (1944).

20. M. K. Kim, D. K. Yi and U. Paik, Langmuir 26, 7552 (2010).

21. K. H. Cheong, D. K. Yi, J.-G. Lee, J.-M. Park, M. J. Kim, J. B. Edel and C. Ko, Lab on a Chip 8, 810 (2009).

22. D.-Y. Khang, H. Jiang, Y. Huang and J. A. Rogers, Science 311, 208 (2006).

23. D.-Y. Khang, J. Xiao, C. Kocabas, S. MacLaren, J. Banks, H. Jiang, Y. Huang and J. A. Rogers, Nano Lett. 8, 124 (2008).

24. Y. Xia and G. M. Whitesides, Soft Lithography, Angew. Chem. Int. Ed. 37, 551 (1998).

25. N. Bowden, S. Brittain, A. G. Evans, J. W. Hutchinson and G. M. Whitesides, Science 393, 146 (1998).

26. W. T. S. Huck, N. Bowden, P. Onck, T. Pardoen, J. W. Hutchinson and G. M. Whitesides, Langmuir 16, 3497 (2000).

27. M. H. Kim, J. Y. Choi, H. K. Choi, S. M. Yoo, O. O. Park, D. K. Yi, S. J. Choi and H. J. Shin, $A d v$. Mater. 20, 457 (2008).

28. D. K. Yi, J-.H. Lee, J. A. Rogers and U. Paik, Appl. Phys. Lett. 94, 084104 (2009).

29. Y. B. Pyun, J. Yi, D. H. Lee, K. S. Son, G. Liu, D. K. Yi, U. Paik and W. I. Park, J. Mater. Chem. 20, 5136 (2010).

30. Y. Zhu, M. Ramasamy and D. K. Yi, ACS Appl. Mater. Interfaces 6, 15078 (2014).

31. Ned Bowden, Wilhelm T. S. Huck, Kateri E. Paul and George M. Whitesides, Appl. Phys. Lett. 75, 2557 (1999).

32. Y. Li, J. Q. Pham, K. P. Johnston and P. F. Green, Langmuir 23, 9785 (2007). 


\section{Supplementary Information}

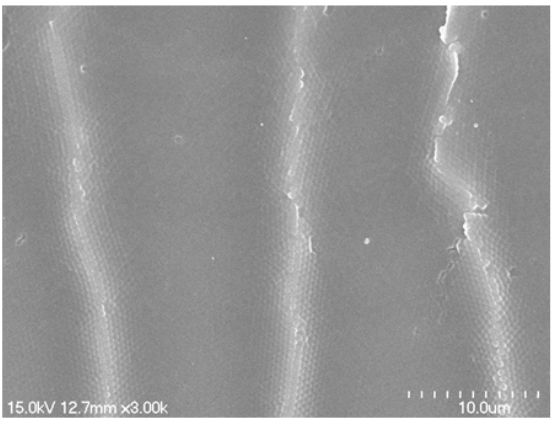

Top view; polystyrene surface

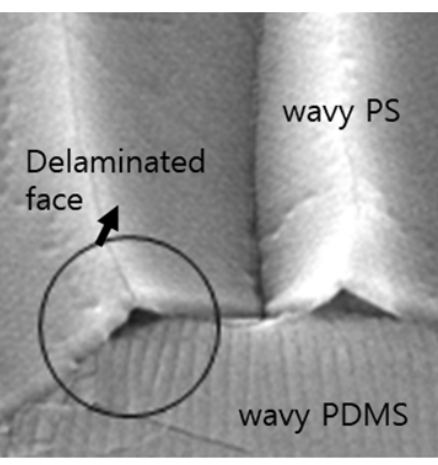

Cross-section view

Fig. S1. SEM image of wavy PS onto the wavy PDMS.

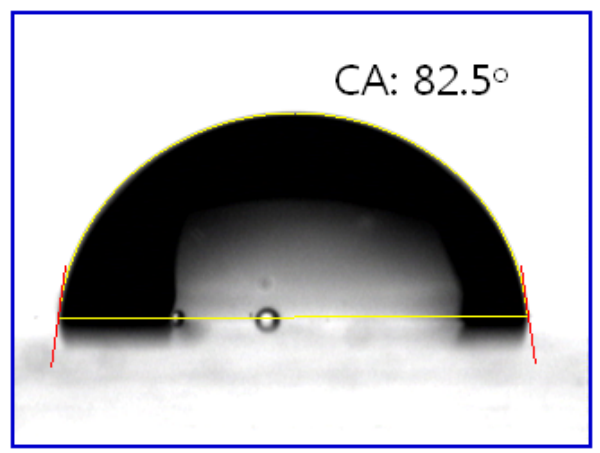

Fig. S2. Water droplet contact angle of nonstrained flat polystyrene film, $\sim 83^{\circ}$. 